\title{
Mapping the Dark Energy with Varying Alpha
}

\author{
David Parkinson ${ }^{\dagger}$, Bruce A. Bassett ${ }^{\dagger}$ and John D. Barrow* \\ ${ }^{\dagger}$ Institute of Cosmology and Gravitation, University of Portsmouth, Portsmouth PO1 2EG, UK \\ * DAMTP, Centre for Mathematical Sciences, University of Cambridge, Cambridge, CB3 OWA, UK
}

(October 30, 2018)

\begin{abstract}
Cosmological dark energy is a natural source of variation of the fine structure constant. Using a model-independent approach we show that once general assumptions about the alpha-varying interactions are made, astronomical probes of its variation constrain the dark energy equation of state today to satisfy $-1<w_{f}<-0.96$ at 3 -sigma and significantly disfavour late-time changes in the equation of state. We show how dark-energy-induced spatial perturbations of alpha are linked to violations of the Equivalence Principle and are thus negligible at low-redshift, in stark contrast to the BSBM theories. This provides a new test of dark energy as the source of alpha variation.
\end{abstract}

The universe appears to be accelerating in response to a dark energy whose identity remains a mystery. We show how the current astronomical data supporting a $5 \sigma$ detection of time variation in the fine-structure 'constant', $\alpha=e^{2} / \hbar c$, at redshifts $0.2<z<3.7[1,2]$ can provide direct evidence distinguishing a dynamical dark energy from a cosmological constant, which is very challenging for standard cosmological tests, e.g. [3]. Unlike cosmological tests which depend on the integrated properties of the dark energy, measurement of $\frac{\Delta \alpha(z)}{\alpha} \equiv \frac{\alpha(z)-\alpha(0)}{\alpha(0)}$ would allow us to map the dark energy as a function of $z$, see also refs. [4-6].

Mapping the dark energy - We will consider a variation of $\alpha$ generated by a non-trivial gauge kinetic function (GKF), $Z_{F}(\phi)$, leading to an electromagnetic lagrangian $\frac{1}{4} Z_{F}(\phi) F_{\mu \nu} F^{\mu \nu}$, where $\phi$ is some scalar field and $F_{\mu \nu}$ the usual Maxwell tensor. (We expect $Z_{F} \neq 1$ in string theory through the coupling to the dilaton [7] or through non-renormalisable interactions in supergravity [8]) In this case $\alpha(z) \propto Z_{F}(\phi)^{-1}$ so if we know $Z_{F}$ and $\Delta \alpha(z) / \alpha$ precisely we can deduce $\phi(z)$.

Is it natural for $\phi$ to be the dark energy? The interaction $Z_{F}(\phi) F^{2}$ is not renormalisable in $4 \mathrm{~d}$ and hence $Z_{F}=Z_{F}(\phi / M)$ where $M$ is the mass scale at which the effective theory description (usually supergravity) breaks down. If we assume $M$ is the Planck mass, $M_{p l} \sim 10{ }^{19} \mathrm{GeV}$, only very large expectation values of $\phi$ will lead to measurable effects. This is what happens in many dark-energy models that typically require large field values, $\phi / M_{p l}=O(1)$ today to satisfy the slow-roll condition, $M_{p l}^{2} V_{\phi \phi}<V$, on the potential $V(\phi)$ needed for an accelerating universe. We will show, using a model-independent approach, how one can constrain the dark-energy's equation of state. Note that such models for varying $\alpha$ differ from those of Bekenstein-SandvikBarrow-Magueijo (BSBM) [9] in which $\alpha$-variations are driven by coupling to the charged non-relativistic matter alone and so the associated scalar field cannot be the dark energy.

We can also consider spatial variation of $\alpha[10,2]$. Dark energy should cluster on scales larger than its Compton wavelength $\lambda_{c} \propto V_{\phi \phi}^{-1 / 2}$, which exceeds $100 M p c$ in standard quintessence models [11], although in more exotic models this need not be true [12]. A map of the spatial variation of $\alpha$ can potentially probe both the power spectrum and Compton wavelength of fluctuations in the dark energy. Consider the spatial fluctuation $\delta \alpha \equiv \alpha\left(x_{\mu}\right)-\bar{\alpha}$. Quantities with an overbar are evaluated in the background cosmology at the same event. To first order in $\delta \phi$ we have

$$
\frac{\delta \alpha(\mathbf{x}, t)}{\alpha}=\left(\left.\partial_{\phi} \ln \alpha\right|_{\phi}\right) \delta \phi .
$$

Evaluated today, this is directly related to Weak Equivalence Principle (WEP) constraints [13] on varying- $\alpha$ theories which, assuming large $\lambda_{c}$ so that we may use the background solution for $\phi$ even in the nonlinear regime*, imply $\delta \alpha /\left.\alpha\right|_{0} \leq 10^{-5} \delta \phi / M_{p l}$, today. Since we expect $\delta \phi \ll M_{p l}$, spatial fluctuations of $\alpha$ should be negligible at low $z$ if dark energy (or any light scalar field) is the source for the time-variation of $\alpha$. This is in agreement with current data [2]. But at high $z$ the spatial fluctuations of $\alpha$ could be significant. Eq. (1) implies that the power spectrum of $\alpha$ fluctuations, $P_{\delta \alpha}(k) \equiv \frac{k^{3}}{2 \pi^{2}}\left|\delta \alpha_{k}\right|^{2}$ is anti-correlated with the spectrum of dark-energy fluctuations, $P_{\delta \phi}(k)$. Their ratio, which we call the $\alpha$-bias $b_{\alpha}$, will in general be time-dependent with potentially interesting implications for the CMB [16].

$Z_{F}$ degeneracy - Our discussion has been predicated on the (unreasonable) assumption that we know the function $Z_{F}$. A standard approach [5] is to assume slow roll for $\phi$ in some potential $V(\phi)$ and expand $Z_{F}$ around $\phi_{0}$, the value of $\phi$ today (see for example ref. [6]). The leading term gives $\Delta \alpha(z) / \alpha \propto \Delta \phi / M_{p l}$. However this is overly restrictive since $Z_{F}$ may not be analytic in $\phi$, the dark energy may not derive from a slowly-rolling scalar field [15],

\footnotetext{
${ }^{*}$ By contrast, in BSBM varying- $\alpha$ theories spatial variation in $\alpha$ is strong because it is driven by gradients in electromagnetically-charged matter, removing the power of local constraints [14].
} 

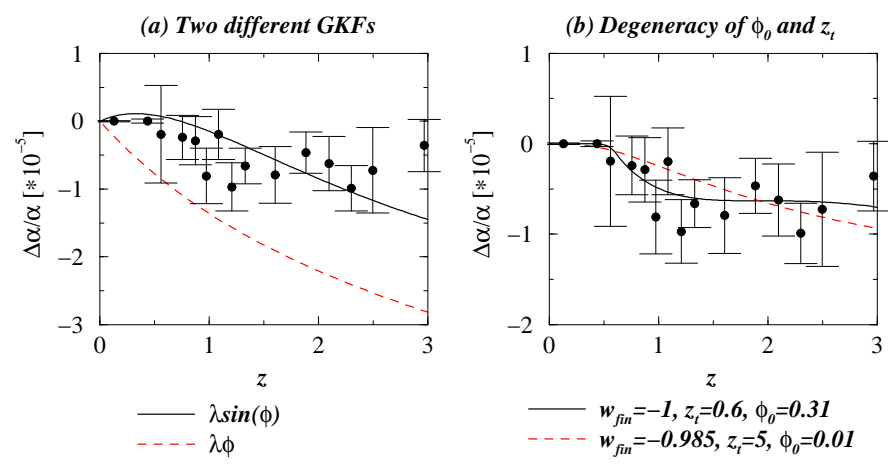

FIG. 1. Degeneracies:

(a) $\Delta \alpha(z) / \alpha$ for the same dark-energy model with two different choices of $Z_{F}$. Note that $Z_{F}=\lambda \sin \phi$ allows both positive and negative values of $\Delta \alpha(z) / \alpha$. (b) The $z_{t}-\phi_{0} / M$ degeneracy in fitting the $\Delta \alpha(z) / \alpha$ data. Both models have almost the same $\chi^{2}$ value. The WEP data breaks this degeneracy.

or $Z_{F}$ may change very rapidly with $\phi$, e.g. $Z_{F} \propto e^{\phi}$ so even if $\phi \sim \ln (t), \Delta \alpha(z) / \alpha$ would exhibit power-law variation with redshift. Hence there is a perfect degeneracy between $Z_{F}$ and the dark-energy dynamics, shown in Fig (1a) by plotting $\Delta \alpha(z) / \alpha$ for the same dark-energy model with two very different GKFs: $Z_{F}(\phi)=\lambda \phi$ and $\lambda \sin (\phi)$. If the resulting curves were assumed to arise from the same GKF, they would have been interpreted as coming from two completely different dark-energy models.

To further illustrate this degeneracy, consider the left branch of the Oklo analysis of the neutron capture resonance [19] which suggests $\Delta \alpha / \alpha=(0.88 \pm 0.07) \times 10^{-7}$. This corresponds to a larger $\alpha$ at $z=0.13$, in contrast to the Webb et al data which strongly suggests $\alpha$ was smaller at $z>0.5$ with $\Delta \alpha / \alpha=-(0.543 \pm 0.116) \times 10^{-5}$, $[2]$.

It has been claimed that this is inconsistent with a slowly-rolling quintessence origin; true if $Z_{F}$ is monotonic in $\phi$. However, a GKF such as $Z_{F}(\phi)=\lambda \sin (\phi)$ can give both positive and negative $\frac{\Delta \alpha(z)}{\alpha}$ and match the apparently inconsistent data with monotonic $\phi(z)$, as shown in Fig. 1(a). In fact, given any data $\Delta \alpha(z) / \alpha$ and any smooth $\phi(z)$ we can reconstruct a corresponding GKF which will exactly fit the data by defining the GKF to be $Z_{F}(\phi(z))=\alpha_{*} / \alpha(z)-1$ where $\alpha_{*} \simeq\left(1+Z_{F}\left(\phi_{0}\right)\right) / 137$ normalises $\alpha(z)$.

Dark energy can induce variation of $\alpha$ in other ways. We can choose a tensor, $g_{E M}^{\mu \nu}$, to raise and lower indices on $F_{\mu \nu}$ that differs from the spacetime metric $g^{\mu \nu}$ used to form the Ricci scalar $R$ [17]. A simple example is

$$
g_{E M}^{\mu \nu}=g^{\mu \nu}\left(1+\frac{(\nabla \phi)^{n}}{M^{2 n+2}} \nabla^{\mu} \phi \nabla^{\nu} \phi\right),
$$

where $\nabla^{\mu}$ is the covariant derivative w.r.t. $g^{\mu \nu}$. This yields $Z_{F}=Z_{F}\left(\left|\nabla_{\mu} \phi\right| / M^{2}\right)$, so $\Delta \alpha / \alpha \sim(H / M)$. If $M=M_{p l}$ such interactions are negligible today; but if $M \sim 10^{-3} \mathrm{eV}$, the models can easily fit the data at $z \leq 3.5$; however, if $\phi$ is a tracker field then $\dot{\phi} \rightarrow \infty$ as $z \rightarrow \infty$. If $Z_{F}(\phi) \propto 1+\left(\dot{\phi} / M^{2}\right)^{n}$ for any $n$, then $\left|Z_{F}(\infty)\right| \rightarrow \infty$ and $\alpha(z) \rightarrow 0$ rapidly. In fact, we have found that models of this form that match the data at $z<3$ but strongly violate the nucleosynthesis constraint $\left|\frac{\Delta \alpha}{\alpha}\left(10^{10}\right)\right|<0.1[18]$. This can be avoided by choosing a GKF with $Z_{F}(\dot{\phi} \rightarrow \infty)=$ constant, or a model with bounded $|\dot{\phi}|$, as in condensation models of dark energy $[12]$, but this goes beyond the scope of the paper. ${ }^{\dagger}$. This shows the importance of theoretical input into choosing $Z_{F}$ and the need for general parametrisations of $Z_{F}$. Below we investigate to what extent we can expect to distinguish between different $Z_{F}$ and different dark energy equations of state.

Consistency - Before we discuss mapping of the dark energy we address in more detail the suggestions in the literature (e.g. [4,20]) that it is difficult, or even impossible, for a slowly-rolling scalar field to match both null geonuclear data $[19,25]$ at $z<0.5$ and the quasar data at $z>0.5$ in $Z_{F}$ theories (it is easier in BSBM theories because there is strong spatial variation [14]). To quantify the extent to which dark energy can match the data we use model-independent approach [12,21-23] which uses $w(z) \equiv p_{\phi} / \rho_{\phi}$ instead of choosing a particular potential. An expedient choice is

$$
w(z)=w_{f}\left[1+\exp \left(\left(\left(z-z_{t}\right) / \Delta\right)\right]^{-1},\right.
$$

so that $w(z \rightarrow \infty)=0 ; w_{f}$ is constant; $z_{t}$ is the redshift at which a transition to dark-energy domination occurs, while the constant $\Delta$ controls its width. This form accurately describes most quintessence models and some exotic alternatives such as vacuum metamorphosis [15]. We fix $\Delta=z_{t} / 30$ as in $[21,12]$ so $w(0)=w_{f}$, and perform a likelihood analysis over the free dark-energy parameters $\left(w_{f}, z_{t}\right)$ and the parameters of the GKF. We have already seen that if $Z_{F}$ is completely free one can match any data with any dark-energy dynamics. Therefore, we need theoretical input or a general parametrisation of the GKF. In the spirit of the model-independent approach we consider an expansion of the GKF in the form:

$$
Z_{F}=1-\Gamma_{n} \varphi^{n}, \quad \Gamma_{n} \equiv \beta_{n}\left(\frac{\phi_{0}}{M}\right)^{n}
$$

where $\varphi(z) \equiv \phi(z) / \phi_{0}$ and $\Gamma_{n}>0$ are dimensionless couplings. We limit ourselves to $n \leq 4$ for computational reasons and consider each $n$ separately. This choice of $Z_{F}(\phi)$ expands our set of parameters to $\left(w_{f}, z_{t} ; \beta, \frac{\phi_{0}}{M}, n\right)$.

\footnotetext{
${ }^{\dagger}$ Another example, the 1-loop QED lagrangian in curved spacetime, [24] yields a GKF of the form$$
Z_{F}=1+a R^{2}+b R_{\mu \nu} R^{\mu \nu}+c R_{\mu \nu \gamma \delta} R^{\mu \nu \gamma \delta},
$$

where $a, b, c$ are constants and $R^{\mu \nu \gamma \delta}$ is the Riemann tensor. However, these terms are Planck suppressed and are not expected to be influential today.
} 


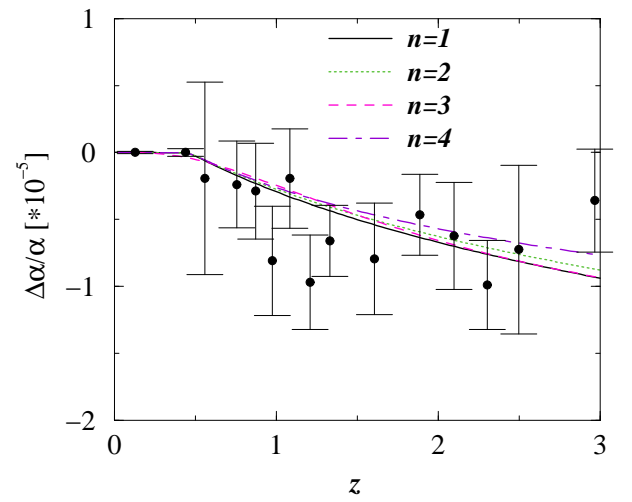

FIG. 2. The individual best-fits for each value of $n$. The difference in $\Delta \alpha / \alpha$ between the models for $z \leq 3$ is less than $2 \times 10^{-6}$ and yet Figure (3) shows that the combined data strongly favour $n=3$. This reflects the highly non-Gaussian nature of the $\chi^{2}$ hypersurfaces.

The best-fits for each $n$, varying the remaining parameters, are shown in Fig. (2).

Our first $\Delta \alpha(z) / \alpha$ data set is $\{\mathrm{QSO}\}$, the quasar absorption data $[1,2]$ providing the evidence for timevariation of $\alpha$. The second set of data is $\{\mathrm{QSO}+$ Oklo $+\operatorname{Re}\} . \quad\{$ Oklo $\}$ is the null branch of the Oklo data $\left(\left|\frac{\Delta \alpha}{\alpha}(z=0.13)\right| \leq 10^{-7}\right)[19] ;\{\operatorname{Re}\}$ is the from the Os/Re ratio from radioactive decay ${ }^{187} \mathrm{Re} \rightarrow^{187} \mathrm{Os}$ in iron meteorites, $[25]\left(\left|\frac{\Delta \alpha}{\alpha}(z=0.44)\right|<3 \times 10^{-7}\right)$ close to the lowest redshift quasars. Last, we add composition-dependent WEP constraints on the difference in the acceleration, $a$, of different bodies $A$ and $B, \eta \equiv(\Delta a / a)_{A B} \leq 10^{-12}$ $[13,4]$ that arises because of their different numbers of charged nucleons. Allowing for gauge coupling unification, $\eta \propto(d \ln \alpha / d \phi)^{2}[7]$ (and note eq. (1)) and this translates into the bound

$$
\frac{n \Gamma_{n}}{\left(1-\Gamma_{n}\right)}\left[\frac{M}{\phi_{0}}\right]<10^{-5} \frac{M}{M_{p l}}
$$

today.

The best-fit reduced- $\chi^{2}$ for the three data sets are 4.7/8, 4.8/10 and 10.4/11 respectively. Adding the WEP constraint clearly has the largest effect but the $\chi^{2}$ values show that general dark-energy models are compatible with the current $\alpha(z)$ data.

Closing the circle - Is it possible to gain information about $Z_{F}(\phi)$ and the dark energy simultaneously? To answer this question we compute the 1-d likelihoods for each one of the parameters in the set $\left(w_{f}, z_{t} ; \beta, \frac{\phi_{0}}{M}, n\right)$ by marginalising over the remaining parameters on a grid consisting of 2.3 million points using the following precise expression for $\Delta \alpha(z) / \alpha$ :

$$
\frac{\Delta \alpha(z)}{\alpha}=-\Gamma_{n} \frac{1-\varphi^{n}(z)}{1-\Gamma_{n} \varphi^{n}(z)} .
$$

Our results, are shown in Fig. (3).
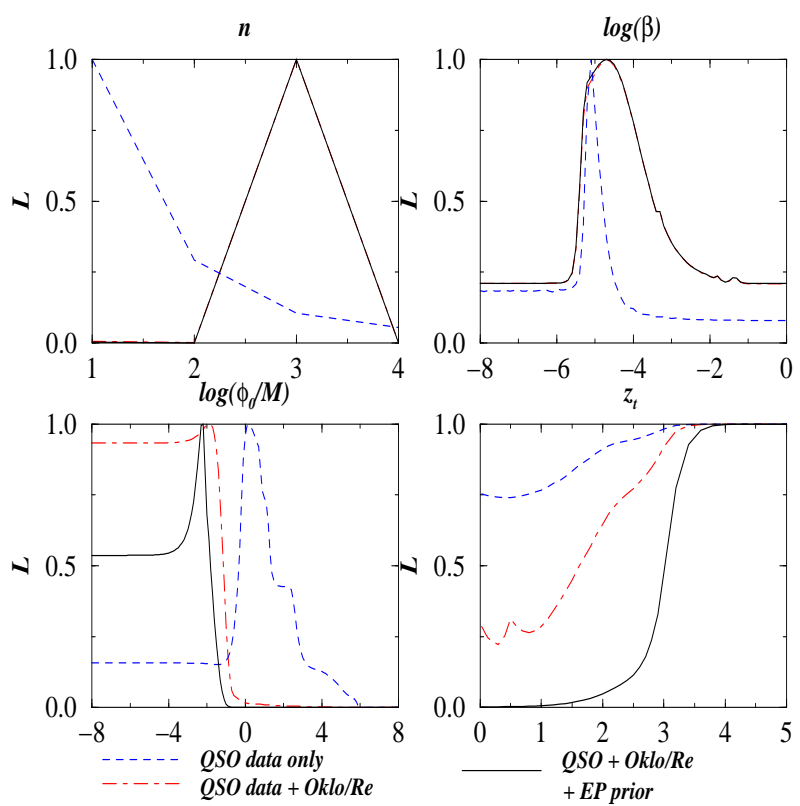

FIG. 3. The marginalised $1 \mathrm{~d}-$-likelihoods for $n, \beta, \phi_{0} / M$ and $z_{t}$ for each of the three combinations of data sets. The curves represent the final likelihoods including all the data. Note how the data strongly favour $n=3$ and how the WEP data selects $\phi_{0} / M \simeq 10^{-2}$ as the most favoured value.

In particular, a dimensionless coupling $\beta \sim 10^{-3}-10^{-5}$ is preferred by all the data. The role of $n$ is interesting. While for each $n$ it is possible to find sets of parameters with nearly equal $\chi^{2}$, Fig. (2), the marginalised likelihood for $n$ is selective, Fig.(3). This reflects the fact that the likelihood hypersurface is highly non-Gaussian, with a large number of nearly degenerate minima. However the most likely values of our parameters depend on the data set used, casting some doubt on the maturity of current data. Considering only the $\{\mathrm{QSO}\}$ data set, $n=1$ is preferred. However, when the low-redshift $\{$ Oklo/Re $\}$ and WEP constraints are added then $n=3$ is significantly favoured, which is a promising sign for future determination of the GKF. If we consider only the $\{\mathrm{QSO}+\mathrm{Oklo}+\mathrm{Re}\}$ data, there is an interesting degeneracy between dark-energy models with low (high) $z_{t}$ and high (low) values of $\phi_{0} / M$. This is illustrated in Fig. (1b) by two good fits to the data with parameter values $\left(z_{t}, \phi_{0} / M\right)=\left(5.0,10^{-2}\right)$ and $\left(0.6,10^{0.5}\right)$ respectively.

In order to map the dark energy we calculate $\phi(z)=$ $\phi_{0}-\int_{0}^{z} \dot{\phi}(z) d z /(H(1+z))$ where $\dot{\phi}^{2}(z)=(1+w(z)) \rho_{\phi}$ and $\rho_{\phi}(z)=\rho_{0} \exp \left(3 \int(1+w) d z /(1+z)\right)[21,12]$. We set $\Omega_{\phi}=$ 0.7 today. These equations imply that as $w_{f} \rightarrow-1$ and $\Delta \alpha / \alpha \rightarrow 0$ for $z<z_{t}$. Clearly, the tighter the null results on $\Delta \alpha$ at low redshift become, the closer $w_{f}$ is pushed to -1 . This can be clearly seen in the likelihood curve for $w_{f}$ in Fig. (4) when the $\{$ Oklo + Re $\}$ and WEP data are added. The WEP constraint is particularly useful since it breaks the degeneracy between $\beta, \phi_{0}$ and $M$ in eq. (7) which only depends on the combination $\Gamma_{n}$. The 
constraint is weakest if we choose $M=M_{p l}$, as we do.

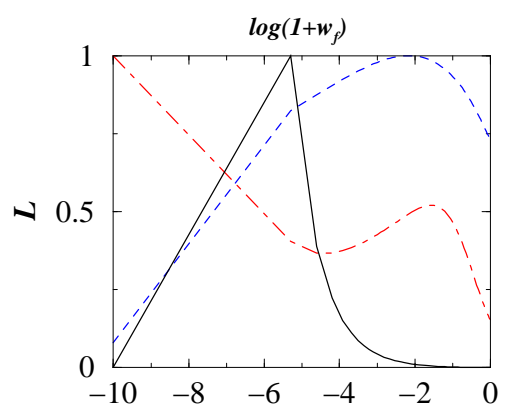

FIG. 4. The 1-d likelihood for $\log \left(1+w_{f}\right)$. The dashed line corresponds to the quasar data, the dot-dashed line to the quasar+Oklo+Re-Os data, and the solid line to all the data (including the WEP violation constraint). The full data set gives $-1<w_{f}<-0.96$ at $3 \sigma$.

The complete set of data favour both $w_{f}$ close to -1 and $z_{t}>2.5$; see Figs (4) \& (3). \{Oklo + Re $\}$ favours $\dot{\phi}(z<0.45)=0$ and hence $w_{f} \simeq-1$. Adding WEP restricts $-1<w_{f}<-0.96$ at $3 \sigma$ with $w_{f}=-1$ significantly disfavoured, see Fig. (4). This is because $w_{f}=-1$ would imply $\Delta \alpha(z) / \alpha=0$ for $z<z_{t}$, and to match the quasar data would then require $z_{t}<0.5$, inconsistent with the likelihood curve for $z_{t}$. Small values of $z_{t}$ values are disfavoured because, as shown in Fig. (1), there is a $z_{t}-\left(\phi_{0} / M\right)$ degeneracy in matching the quasar data. Models with small $z_{t}$ require large values of $\phi_{0} / M$, but large $\phi_{0} / M$ is disfavoured by the Oklo/Re data (Fig 3). Conversely, very small $\phi_{0} / M$ are disfavoured (at constant $\left.\Gamma_{n}\right)$ by the WEP data, eq. (6).

For $n=1$ (the standard dimension-5 case $[4,5]$ ) the constraint (6) yields $\beta_{1}<10^{-5}\left(M / M_{p l}\right)$, while for $n \geq 2$ it is a joint constraint on $\Gamma_{n}$ and $\phi_{0} / M$. In the next decade the STEP [26], GG [27] and MICROSCOPE [28] experiments promise sensitivities up to $\eta<10^{-18}$ and hence a null detection would imply $d \ln \alpha / d \phi<10^{-8}$ today, virtually ruling out $n=1$. To investigate potential future constraints we can impose this expected bound on our best-fits for each $n$. The resulting reduced $-\chi^{2} \mathrm{~s}$ all exceed $38 / 11$ and would all be excluded by this future data.

In summary, by using a model-independent approach to the dark energy, we have shown that the current constraints on $\Delta \alpha(z) / \alpha$ can be well-matched by dark-energy models. For a large family of varying- $\alpha$ theories, we have shown how spatial fluctuations in $\alpha$ are correlated with fluctuations of the dark energy but need to be negligibly small at low-redshifts due to Weak Equivalence Principle constraints, so providing a clear test of the scenario. We have pointed out that the gauge kinetic function, $Z_{F}$, responsible for the variation of $\alpha$ is completely degenerate with the dark energy dynamics so that any dynamical dark-energy model can be made to fit the $\frac{\Delta \alpha(z)}{\alpha}$ data. Nevertheless, with a reasonable parametrisation of $Z_{F}$ the current data already yield interesting constraints on $Z_{F}$. When the WEP constraint is added the current equation of state is forced to lie in the range $-1<w_{f}<-0.96$ at $3 \sigma$, the tightest constraints on the dark energy yet found. This is unlikely to change much if one varies other cosmic parameters. Late-time change in $w(z)$ at $z<2$ and $\frac{\phi_{0}}{M}>10^{-1}$ are also strongly disfavoured, in contrast to standard CMB/LSS results $[21,12]$, raising the possibility that a complete combined analysis will rule out most dark-energy models or even perhaps the paradigm of dark-energy-induced variation of $\alpha$.

Finally, we note that the GKFs we have considered break the conformal invariance of the Maxwell equations and may thus allow the seeding of magnetic fields during inflation. This would provide an interesting way of linking the early universe to observations in the present epoch.

We thank John Webb for supplying us with the QSO data, and Federico Piazza, Rob Crittenden, Haim Goldberg, Sujata Gupta, Martin Kunz, Andrew Liddle, André Lukas and Michael Murphy for useful discussions and comments.

[1] J. K. Webb et al, Phys. Rev. Lett. 87, 091301 (2001); J. K. Webb et al, Phys. Rev. Lett. 82, 884 (1999)

[2] M. T. Murphy, J. K. Webb, V. V. Flambaum, astro$\mathrm{ph} / 0306483$

[3] I. Maor, R. Brustein, P. J. Steinhardt, Phys. Rev. Lett. 86, 6 (2001); P.S. Corasaniti et al, Phys. Rev. Lett. 90, 091303 (2003)

[4] T. Chiba, K. Kohri, Prog. Theor. Phys. 107, 631 (2002)

[5] J.D. Bekenstein, Phys. Rev. D 25, 1527 (1982); H. Sandvik, J.D. Barrow and J. Magueijo, Phys. Rev. Lett. 88, 031302 (2002); C. Wetterich, hep-ph/0203266 and astroph/0302116, Z. Chacko, C. Grojean \& M. Perelstein, hepph/0204142; A. Kostelecky, R. Lehnert, M. Perry, astro$\mathrm{ph} / 0212003$

[6] L. Anchordoqui and H. Goldberg, hep-ph/0306084

[7] T. Damour, F. Piazza, G. Veneziano, Phys. Rev. Lett. 89081601 (2002) and Phys. Rev. D 66, 046007 (2002)

[8] S. M. Carroll, Phys. Rev. Lett. 813067 (1998)

[9] J.D. Barrow, H. Sandvik and J. Magueijo, Phys. Rev. D 65, 065504 (2002) and 123501 (2002)

[10] J.D. Barrow and D.F. Mota, Class. Quantum Grav. 20, 2045 (2003); J.D. Barrow and C. O'Toole, MNRAS 322, 585 (2001)

[11] C.-P. Ma et al, Ap.J. 521, L1 (1999)

[12] B. A. Bassett, M. Kunz, D. Parkinson and C. Ungarelli, astro-ph/0211303 (2002)

[13] T. Damour and A. Polyakov, Nucl. Phys. B 423, 532 (1994); ; J. Magueijo, J.D. Barrow and H. Sandvik, Phys Lett. B 549, 284 (2002)

[14] D. F. Mota and J. D. Barrow, astro-ph/0306047

[15] L. Parker and A. Raval, Phys. Rev. D 62, 083503 and 
Phys. Rev. D 67, 029903 and gr-qc/9908069

[16] K. Sigurdson, A. Kurylov and M. Kamionkowski, astro$\mathrm{ph} / 0306372$

[17] B. A. Bassett, S. Liberati, C. Molina-Paris, M. Visser, Phys.Rev. D62 103518 (2000)

[18] K.M. Nollett and R. Lopez, Phys.Rev. D 66, 063507 (2002)

[19] A. Shlyakhter, Nature 260, 340 (1976); Y. Fujii et al, Nucl. Phys. B 573, 381 (2000); T. Damour and F. Dyson, Nucl. Phys. B 480, 37 (1996)

[20] T. Damour, gr-qc/0306023

[21] B. A. Bassett, M. Kunz, J. Silk and C. Ungarelli, MNRAS 336, 1217 (2002)

[22] P.S. Corasaniti, E.J. Copeland, astro-ph/0205544

[23] E. V. Linder, Phys. Rev. Lett. 90, 091301 (2003)

[24] I.T. Drummond and S.J. Hathrell, Phys. Rev. D22, 343 (1980)

[25] K. A. Olive, M. Pospelov, Phys.Rev. D 65, 085044; K. A. Olive et al, Phys.Rev. D 66045022 (2002)

[26] See http://einstein.Stanford.EDU/STEP/

[27] See http://eotvos.dm.unipi.it/nobili/

[28] See http://www.onera.fr/dmph-en/accelerometre/ 